\title{
MAGNITUD Y FRECUENCIA DEL TRANSPORTE FLUVIAL DE SEDIMENTO EN UNA CUENCA MEDITERRANEA SEMIHUMEDA
}

\author{
Ramón J. BATALLA \& María SALA
}

Grup de Recerca Ambiental Mediterrània, Departament de Geografia Fisica, Universitat de Barcelona, c/ Baldiri i Reixac s/n, 08028 Barcelona

\begin{abstract}
RESUMEN.- Este trabajo examina la magnitud y la frecuencia del transporte de sedimento (material disuelto, sedimento en suspensión y carga de fondo) en la cuenca de la riera de Arbúcies (Cordilleras Costeras Catalanas), a partir de los caudales diarios del periodo 1967-1992 y de las muestras de agua y sedimento obtenidas durante los años 1991 y 1992. Los resultados indican que el transporte en disolución es más uniforme en el tiempo que el transporte de material sólido, tanto en suspensión como de fondo. Los cálculos muestran que la carga sólida total depende sobretodo de crecidas frecuentes y de magnitud moderada, correspondientes al nivel de cauce lleno (bankfull). Cabe señalar, en este sentido, que los caudales bankfull suceden el $2,2 \%$ del tiempo y transportan el $56 \%$ del sedimento en suspensión y el $31 \%$ de la carga de fondo total anual. El caudal efectivo para el transporte de sedimento en este rio es, por tanto, un suceso relativamente frecuente y de idéntica magnitud al caudal geomorfológicamente dominante o bankfull.
\end{abstract}

ABSTRACT.- This study examines the magnitude and frequency of the sediment transport in the Arbucies drainage basin (Catalan Coastal Ranges). For this purpose, daily discharges for the period 1967-1992 and sediment samples (including dissolved, suspended and bedload) obtained during 1991 and 1992 are taken into account. Results indicate that dissolved load is more constant through time, while solid material transport is more dependent on floodevents, especially those associated with bankfull discharges. Calculations show that sediment yield is dominated by events of high frequency and moderate magnitud associated with the bankfull discharge. Those events occur $2.2 \%$ of the time and carry $56 \%$ of the anual suspended sediment load and $31 \%$ of the anual bedload yield. Effective discharge for bedload transport in the Arbucies river is, therefore, a relatively frequent flood event of identical magnitude to the bankfull (geomorphic dominant) discharge.

RÉSUMÉ.- Ce travail examine l'ampleur et la fréquence du transport de sédiment (matériel dissous, sédiment en suspension et charge de fond) dans le bassin de la "sierra de Arbúcies" (Cordillères Côtières Catalanes), à partir 
des débits journaliers de la période 1967-1992 et des échantillons d'eau et de sédiment obtenus durantles années 1991 et 1992. Les résultats indiquent que le transport en dissolution est plus uniforme dans le temps que le transport de matériel solide, tant en suspension que de fond. Les calculs montrent que la charge solide totale dépend surtout des crues d'eau fréquentes et d'ampleur modérée, correspondantes au niveau du canal plein (bankfull). Ou signale, dans ce sens, que les débits bankfull surviennent pendant $2,2 \%$ du temps et transportent $56 \%$ du sédiment en suspension et $31 \%$ de la charge de fond annuelle totale. Le débit effectif pour le transport de sédiment dans cette rivière est, donc, un évènement relativement fréquent et d'ampleur identique à celle du débit géomorphologiquement dominant ou bankfull

Key-words: Sediment transport, Sediment yield, Sediment budget, Bedload, Bankfull discharge.

\section{Introducción}

Los procesos de erosión, transporte y deposición de sedimento tienen un creciente interés desde el punto de vista de la gestión medio ambiental de cuencas de drenaje. Dentro del campo de la geomorfologia, los trabajos pioneros sobre transporte de sedimento fueron realizados por Leopold \& Maddock (1953), Emmett (1970) y Carson et al. (1973). Son importantes también los estudios posteriores de Walling (1974) y Walling \& Webb (1981). No obstante, este tipo de información no está generalmente disponible en cuencas de drenaje Mediterráneas de la Península lbérica. Cabe mencionar, en este sentido, los trabajos realizados por Sala (1983) y Sala \& Wheeler (1988) en la cuenca del rio Tordera. La información sobre carga de sedimento es muy útil para la evaluación de la producción de sedimento y las tasas de erosión de cuencas de drenaje, así como para conocer la dinámica del sedimento durante las crecidas y para establecer los correspondientes efectos geomorfológicos aguas abajo. Desde un punto de vista técnico y aplicado, esta información puede resultar muy valiosa para la planificación y gestión de los diferentes usos del suelo asi como para diversas aplicaciones en obras de ingenieria civil (construcción de puentes, colmatación de embalses, redes de drenaje urbanas).

La capacidad de un caudal de agua para el transporte de sedimento a lo largo del tiempo viene determinado por dos factores principales: magnitud y frecuencia. Los conceptos de magnitud y frecuencia fueron desarrollados por Wolman \& Miller (1960). Uno de los principios que ellos presentaron es que el caudal efectivo para el transporte de sedimento es un suceso relativamente frecuente pero de poca magnitud. Estos autores sugirieron que en medios húmedos, el caudal efectivo es similar en magnitud y frecuencia al caudal de cauce lleno (bankfull) que es el que controla la morfologia del canal. No obstante, Benson \& Thomas (1966) concluyeron que para muchos rios el caudal efectivo es inferior al caudal bankfull, situándose entre el caudal medio anual y el caudal indicado en las investiga- 
MAGNITUD Y FRECUENCIA DEL TRANSPORTE FLUVIAL DE SEDIMENTO

ciones de Wolman \& Miller. Hasta el momento se han realizado pocos estudios para relacionar los dos conceptos. Pickup \& Warner (1976) encontraron en un pequeño río de gravas cerca de Sydney (Australia), que el caudal efectivo para el transporte de fondo tiene un periodo de retorno inferior que el caudal bankfull. . Andrews (1980) ha demostrado con datos del rio Yampa, Estados Unidos, que para todo el sedimento transportado el caudal efectivo es un suceso frecuente superado entre 1 y 10 veces cada año (0,35-3\% del tiempo). Ashmore \& Day (1988) indicaron que la frecuencia del caudal efectivo está relacionada con la superficie de drenaje de la cuenca. Estas diferencias se reflejan en la forma de la curva de frecuencias de caudales (Pickup \& Warner, 1976).

Desde 1991 se está llevando a cabo un proyecto de investigación en la cuenca Mediterránea subhúmeda de la riera de Arbúcies (Batalla, 1993), cuyo principal objetivo es construir un balance de sedimento, con un especial interés en el estudio de la contribución del transporte de material sólido a la producción de sedimento de la cuenca. Este artículo examina las relaciones entre caudal y concentración de sedimento y analiza la magnitud y la frecuencia de la carga transportada en este río, a partir del cálculo de la producción de sedimento de la cuenca y del análisis de la curva de frecuencias del transporte de material disuelto, en suspensión y de fondo.

\section{Area de estudio}

La riera de Arbúcies es uno de los afluentes principales del rio Tordera. Está situada en la parte norte de las Cordilleras Costero Catalanas, tiene su cabecera en la Cordillera Prelitoral, y drena una superficie de $114 \mathrm{~km}^{2}$ recogiendo las aguas de las vertientes nororientales del macizo del Montseny. El principal material que forma el sustrato de la cuenca es la granodiorita biotítica de grano medio, que representa más del $80 \%$ de la superficie. Los procesos periglaciares que actuaron durante el Pleistoceno en vertientes por encima de los 500 metros sobre el nivel del mar produjeron, mediante la microfracturación, una importante disgregación de la roca. Como resultado de ello, considerables cantidades de material arenoso fueron expuestas a la erosión y movidas hasta los cursos fluviales. Los depósitos cuaternarios constituyen el $10 \%$ de la superficie de la cuenca y se componen de tres niveles de terrazas Holocenas en las cuales el material arenoso y las gravas finas son predominantes. El material del lecho del río está compuesto de arenas y gravas no uniformes con un $D_{50}$ de $2,2 \mathrm{~mm}$ y un $D_{95}$ de $71 \mathrm{~mm}$ Se observan, asimismo, cambios de textura entre el material del lecho del rio y el sedimento de los márgenes del canal: $D_{50}$ de $0,7 \mathrm{~mm}$ y $D_{95}$ de $6 \mathrm{~mm}$.

La precipitación media anual es de $947 \mathrm{~mm}$, con una alta variabilidad interanual (coeficiente de variación de $30,6 \%$ ), y con un ritmo pluviométrico estacional Otoño - Primavera - Invierno-Verano, característico de la vertiente mediterránea oriental (Martín Vide, 1985). La evaporación media anual es 
de $637 \mathrm{~mm}$. El análisis estadístico de los caudales diarios para el período 1967-1992 (Batalla 1993), muestra que hay un flujo continuo de agua el $98 \%$ del tiempo. El caudal medio es de $1,1 \mathrm{~m}^{3} \mathrm{~s}^{-1}$ y el flujo de base medio es de 0,5 $\mathrm{m}^{3} \mathrm{~s}^{-1}$. El indice de variabilidad de la curva de frecuencias de caudales (Fig.1) utilizado por Lane \& Lei (1950) es 0,39. Las crecidas alcanzan los $65 \mathrm{~m}^{3} \mathrm{~s}^{-1}$ (período de retorno de 50 años). El índicè de magnitud de crecidas de Beard (1975) y Baker (1977) de 0,52.

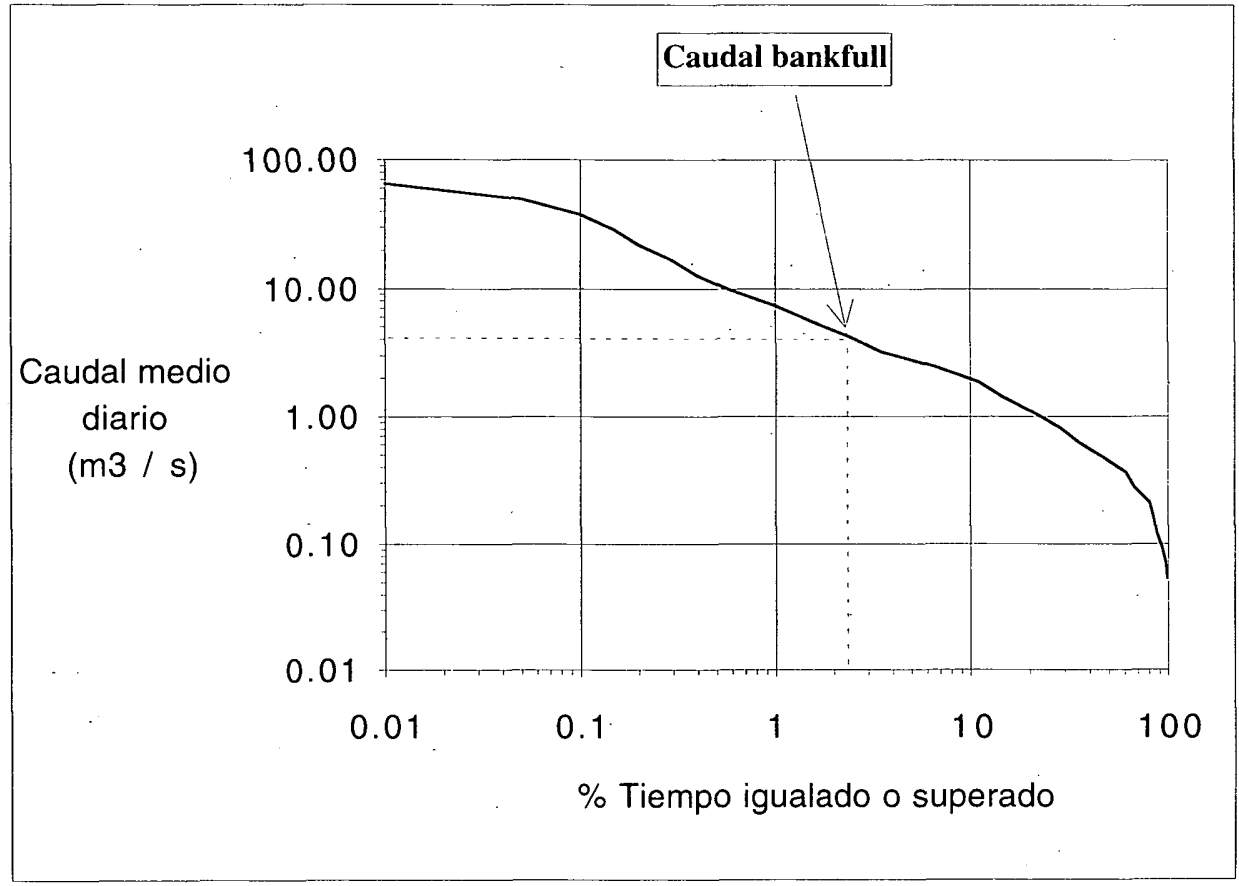

Figura 1. Curva de frecuencias de caudales de la estación n56 (1967-1992), riera de Arbúcies. Se indica el punto medio del caudal de cauce lleno o bankfull. (Curve of frequencies of the discharge in Arbucies river. Bankfull stage is indicated).

El bosque de encinas y robles cubre más del $95 \%$ de la cuenca. En las llanuras aluviales se encuentran plantaciones de chopos para producción de papel. La población se concentra en el pueblo de Arbúcies (3515 habitantes en 1989). Las actividades agricolas se encuentran en clara recesión y se concentran, sobre todo, en los fondos de valle y en las superficies de erosión ( $4 \%$ superficie de la cuenca). 
MAGNITUD Y FRECUENCIA DEL TRANSPORTE FLUVIAL DE SEDIMENTO

\section{Metodología}

Las concentraciones de material en disolución, sedimento en suspensión y carga de fondo se han obtenido en la estación de aforos n56 de la Junta d'Aigües de la Generalitat de Catalunya, a la salida de la cuenca de la riera de Arbúcies, a partir de muestreos semanales y muestreos durante avenidas en 1991 y 1992. Las muestras de 0,5 litros para el material disuelto $y$ en suspensión se han tomado integradas verticalmente mediante un muestreador US DH48 entre $8 \mathrm{~cm}$ del lecho del río y la superficie del agua. El muestreo durante las crecidas se ha realizado mediante un muestreador de nivel de botellas y mediante un tomamuestras automático ISCO-3700. Las tomas manuales integradas de sedimento en suspensión recogidas durante las crecidas se han utilizado para calibrar los muestreadores automático y de nivel instalados en la estación. El transporte de carga de fondo se ha muestreado semanalmente y durante las crecidas mediante un muestreador Helley-Smith de $76 \mathrm{~mm}$. de entrada, red de 0,45 mm. y $15 \mathrm{~kg}$. de capacidad. Las muestras se tomaban integradas en intervalos de 1 metro con una frecuencia de muestreo que oscilaba entre 5 y 10 minutos. Cabe señalar, que para particulas más grandes de $0,50 \mathrm{~mm}$. y más pequeñas de $16 \mathrm{~mm}$., la efectividad de este muestreador llega a alcanzar el 100\%. Para partículas con un tamaño superior a $16 \mathrm{~mm}$. la efectividad en el muestreo desciende hasta un $70 \%$ (Emmett, 1979). Las muestras de carga de fondo tomadas en la riera de Arbúcies no han sido corregidas, ya que la proporción de partículas con un diámetro superior a $16 \mathrm{~mm}$. era a menudo despreciable (< 1\%) y, por tanto, el cálculo final de la carga no se vio afectada por las condiciones del muestreador. El caudal de agua se ha obtenido de manera rutinaria como el producto del área de la sección $\left(\mathrm{m}^{2}\right)$ y la velocidad media del flujo $\left(\mathrm{m} \mathrm{s}^{-1}\right)$ : a) la profundidad del agua y la velocidad medida en intervalos no superiores al $10 \%$ de la anchura del canal ( 5 metros) y b) la velocidad media del flujo tomada a 0,4 de la profundidad del flujo, mediante un molinete hidráulico OTT C-2.

La producción anual de sedimento se ha calculado a partir de la relación estadistica significativa y corregida entre caudal y concentración de sedimento (Ferguson, 1986), aplicada a la curva de frecuencias de caudales obtenida a partir de datos diarios de la riera de Arbúcies para el periodo 1967-1992 (Tabla 1), siguiendo el método propuesto por Piest (1964) y Walling $(1977,1984)$.

\section{Frecuencia y ajuste del canal al caudal de cauce lleno o bankfull}

El nivel del caudal bankfull se ha estimado a partir de criterios topográficos en el campo y sobre evidencias físicas (Batalla \& Sala, 1993), como por ejemplo el limite inferior de la vegetación perenne (Schumm, 1960) y la relación mínima entre anchura y profundidad del canal (Wolman, 1955) que 
PIRINEOS $143-144$

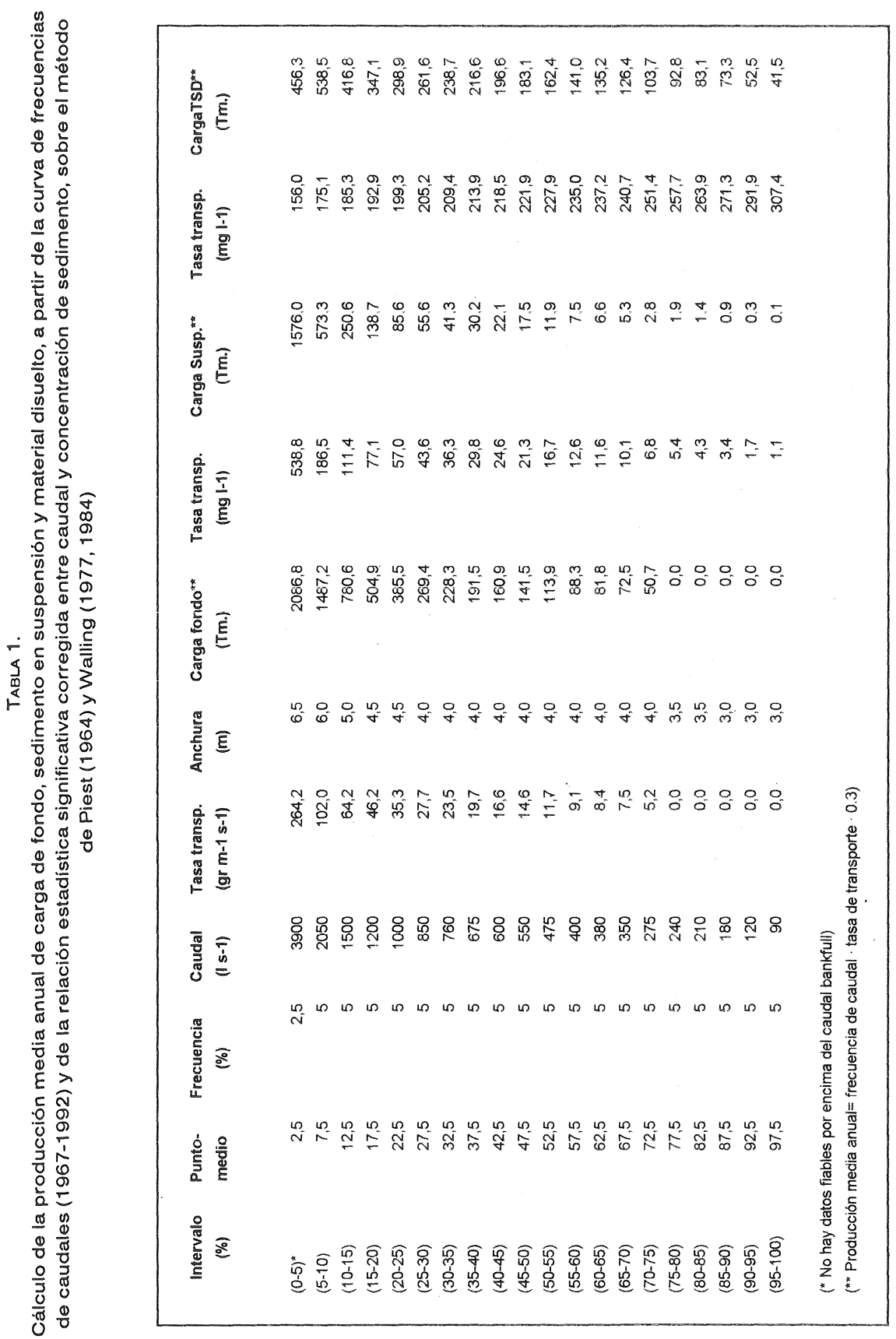

8 
es de 9,3 para la riera de Arbúcies. La curva de frecuencias de caudales (Fig.1) muestra que el caudal que corresponde al nivel bankfull se sitúa alrededor de los $4 \mathrm{~m}^{3} \mathrm{~s}^{-1}$, que es igualado o superado el 2,2\% del tiempo, 10 que significa una mediana de 6-7 dias por año. La anchura y profundidad máximas del cauce para el nivel bankfull son de 7,5 y 0,8 metros respectivamente, y la velocidad media para este caudal es de $1,1 \mathrm{~m}^{3} \mathrm{~s}^{-1}$ (Fig. 2).

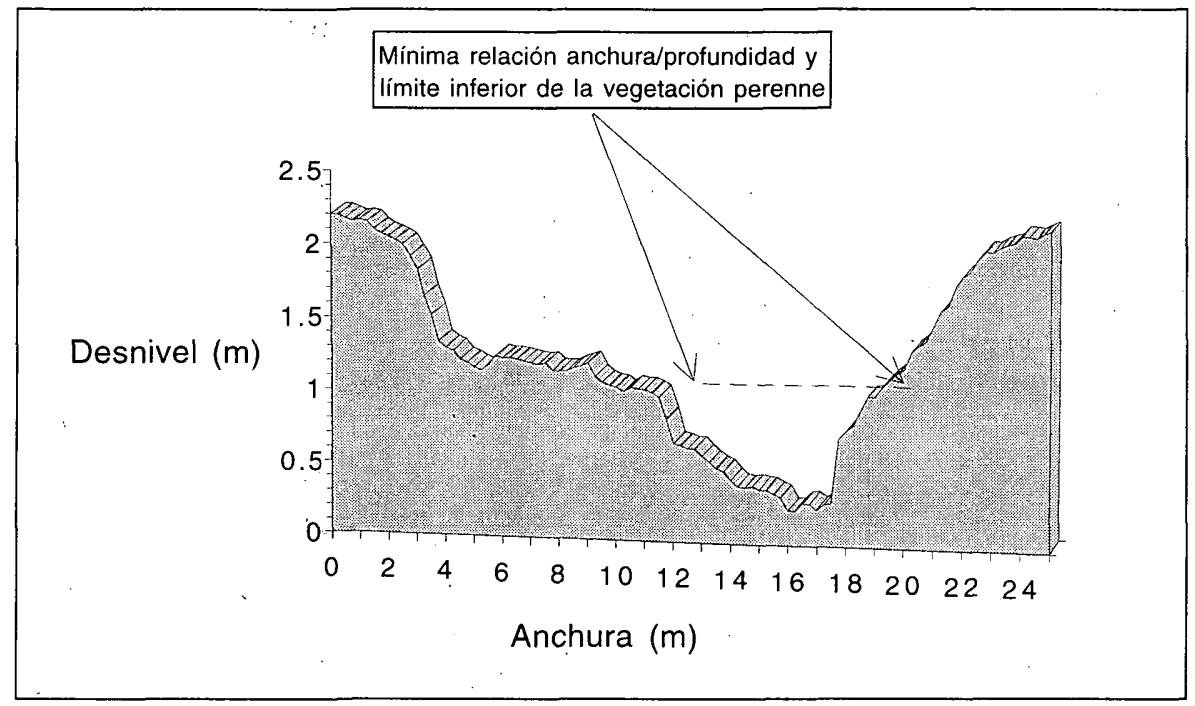

Figura 2. Sección transversal aguas arriba de la estación de aforos. Se indican las evidencias del canal bankfull, como el punto de mínima relación entre anchura y profundidad y limite inferior de la vegetación perenne. (Cross section upstream of the gauging station. Bankfull stage is indicated as the point of minimum relation between width and depth and as the lower limit of perennial vegetation).

Los primeros resultados, obtenidos a partir de la instalación de cadenas magnetizadas durante 1992 aguas arriba de la estación de aforos 56 , indican que durante las crecidas se producen importantes procesos de excavación y deposición de sedimento en el lecho. Para niveles bankfull como mínimo $50 \mathrm{~cm}$ de material es erosionado y sedimentado durante tales crecidas. Es interesante señalar que la dinámica de sedimento observada durante estas crecidas representa tan sólo oscilaciones a corto plazo sobre las condiciones medias del canal, y no por ello se pueden extraer consecuencias sobre la evolución morfológica del valle principal de esta cuenca. 


\section{Resultados}

Los resultados de transporte de sedimento, obtenidos sobre un total de doscientas cuarenta muestras de material disuelto y sedimento en suspensión y setenta y dos de carga de fondo, han permitido establecer la producción media de sedimento de la cuenca y la frecuencia de transporte (Fig 3).

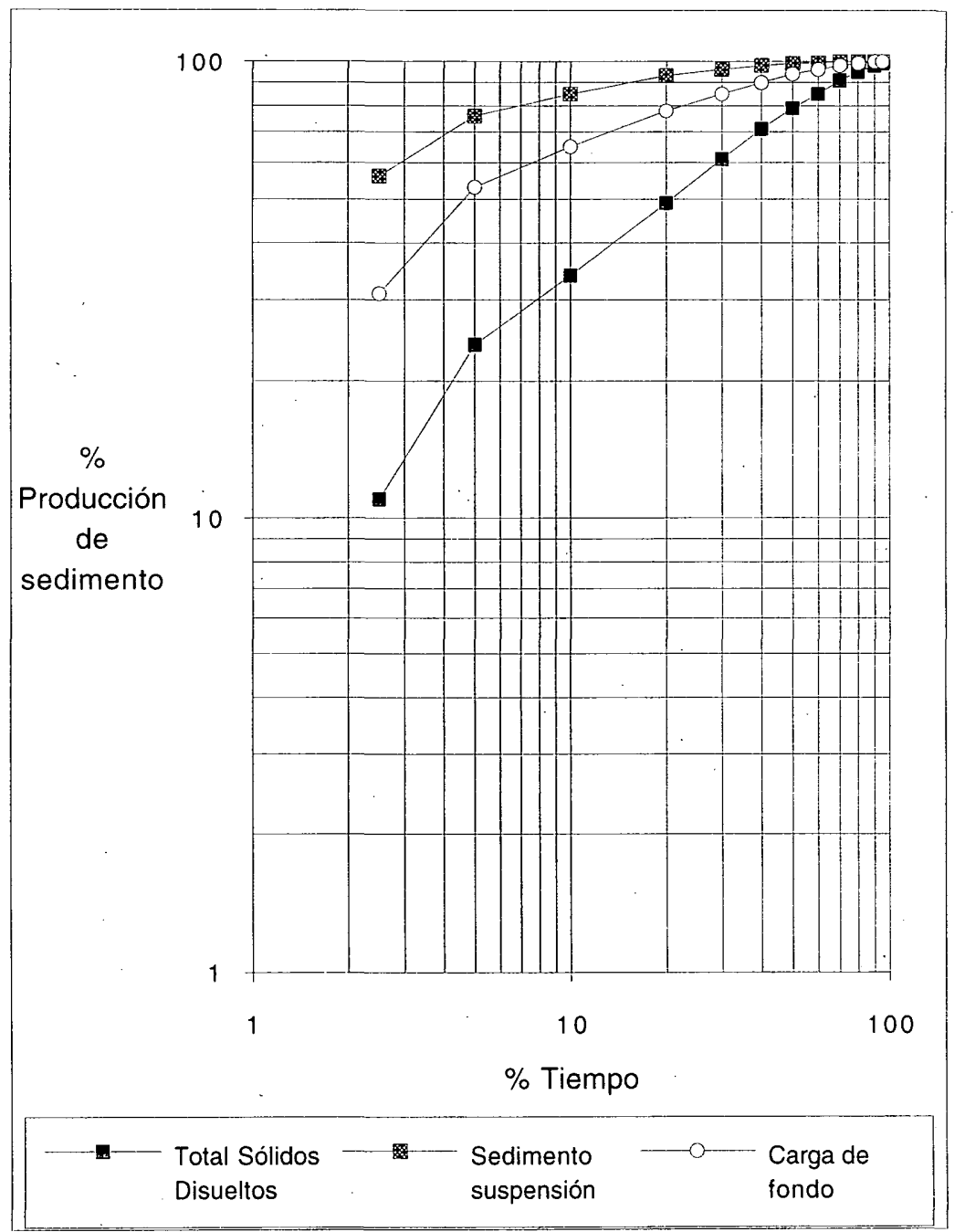

Figura 3. Curva de frecuencias acumuladas para cada una de las modalidades de transporte en la cuenca de la riera de Arbúcies. (Curve of accumulated frequencies for each type of sediment transport in the Arblcies river).

10 


\subsection{Transporte en disolución}

La concentración media del agua de la riera de Arbúcies es de 5,1 meq $\mathrm{I}^{-1}$. Está dominada por $\mathrm{HCO}_{3}^{-}(35,8 \%)$ y Ca ${ }^{++}(24,9 \%)$, seguido por $\mathrm{Na}^{+}(13,0 \%)$, $\mathrm{Mg}^{++}(8,3 \%), \mathrm{Cl}^{-}(6,2 \%), \mathrm{SO}_{4}{ }^{2-}(6,0 \%)$ y $\mathrm{SiO}_{2}(2,4 \%)$, siendo $\mathrm{NO}^{3-}(1,2 \%)$ y K$(1,2 \%)$ los elementos minoritarios. El pH se mantiene constante en todas las muestras con una media de 7,3 y un coeficiente de variación de $2,8 \%$. La conductividad eléctrica media a $25^{\circ} \mathrm{C}$ es de $311,9 \mu \mathrm{S} \mathrm{cm} \mathrm{cm}^{-1}$ con un coeficiente de variación de $20,8 \%$. La relación estadística entre caudal ( $Q$ en I s ${ }^{-1}$ ) y Total de Solidos Disueltos (TSD en $\mathrm{mg} \mathrm{l}^{-1}$ ) se define por la siguiente ecuación significativa $(p<0,01)$ :

$$
\text { TSD }=691 Q^{-0,18} \quad\left(r^{2}=0,78\right) \quad(N=240)
$$

Esta ecuación, juntamente con las entradas atmosféricas obtenidas, ha permitido establecer el balance hidroquímico y la producción total de material disuelto de la cuenca (Batalla \& Sala, 1992). La producción media anual es de $4166 \mathrm{Tm}$., lo que significa una contribución específica del área drenada de $393 \mathrm{~kg} \mathrm{ha} \mathrm{ka}^{-1} \mathrm{an}^{-1}$. La contribución media anual de las sales disueltas en el agua de lluvia es del 15\% (620 Tm.), siendo este aporte especialmente significativo durante las crecidas (superior al $40 \%$ en algunos casos). La contribución de las crecidas a la producción media anual de material disuelto de la cuenca varia entre un $0,5 \%$ y un $3 \%$, siendo función directa de la magnitud de las mismas, es decir del caudal total drenado. La carga de material disuelto representa un porcentaje muy bajo respecto al caudal total drenado durante las crecidas, siendo por término medio no superior al 0,015\% del volumen total exportado.

\subsection{Transporte de material sólido}

El proceso de meteorización de la granodiorita no solamente libera iones sino que además, a causa de la fragmentación de la roca, libera una importante cantidad de material fino y arenoso que es transportado en suspensión o como carga de fondo a lo largo de la red fluvial.

a) Carga de sedimento en suspensión

La composición mineralógica del sedimento en suspensión de la riera de Arbúcies está dominada por los filosilicatos laminares, como el hidróxido de magnesio y la mica biotítica, seguido por diferentes tectosilicatos como el cuarzo y los feldespatos alcalinos. La concentración media de sedimento en suspensión es $191 \mathrm{mg} \mathrm{l}^{-1}$ con un coeficiente de variación del $154,2 \%$. Las concentraciones varian entre $1 \mathrm{mg} \mathrm{l}^{-1}$ durante caudales bajo hasta $2650 \mathrm{mg}$ $\mathrm{I}^{-1}$ obtenido durante las crecidas. La relación estadistica entre caudal ( $Q$ en 
$\left.\right|^{-1}$ ) y concentración de sedimento en suspensión ( $S S$ en $\mathrm{mg} \mathrm{I}^{-1}$ ) es significativa $(p<0,01)$ y se define por la siguiente ecuación:

$$
S S=0,0004 Q^{1,63} \quad\left(r^{2}=0,70\right)
$$

La desviación estadística de la relación debida a la dispersión de puntos ha sido calculada en un $60 \%$. La dispersión de puntos se debe a causas de variabilidad estacional y a fenómenos de histéresis durante las crecidas (Batalla \& Sala, 1994). La producción anual de sedimento en suspensión es de $2829 \mathrm{Tm}$., siendo la contribución específica de $266 \mathrm{~kg} \mathrm{ha}^{-1} \mathrm{an}^{-1}$. La mayoría del sedimento en suspensión es transportado por crecidas de alta frecuencia y moderada magnitud asociadas a caudales del tipo bankfull. Cabe señalar en este sentido que las dos crecidas más importantes de los años 1991 y 1992 con duraciones entre 55 y 89 horas y con periodos de retorno de los picos de las mismas de 1,2 años, contribuyeron con más de $15 \%$ de la producción anual del sedimento transportado en suspensión. El caudal sólido medio drenado durante estas crecidas se situó alrededor del 0,025 del caudal líquido con concentraciones medias de sedimento entre 434 y $730 \mathrm{mg} \mathrm{l}^{-1}$.

\section{b) Carga de fondo}

La tasa media de transporte de fondo obtenida a partir de 72 muestras integradas transversalmente es de $37.5 \mathrm{gr} \mathrm{m}^{-1} \mathrm{~s}^{-1}$ en peso sumergido, con un coeficiente de variación de $178,3 \%$. Las tasas varían entre $1 \mathrm{gr} \mathrm{m}^{-1} \mathrm{~s}^{-1}$ en caudales bajos hasta $280 \mathrm{gr} \mathrm{m}^{-1} \mathrm{~s}^{-1}$ durante las crecidas. La producción de carga de fondo de la cuenca está claramente dominada por el movimiento casi continuo de las particulas de arena en el lecho del rio, a partir de 250 $\mathbf{s}^{-1}$, lo que significa más del $80 \%$ del tiempo y con una tensión de corte inicial de $7,4 \mathrm{~N} \mathrm{~m}^{-2}$. La relación estadística entre caudal y carga de fondo ( $i_{b}$ en $g r$ $\mathrm{m}^{-1} \mathrm{~s}^{-1}$ de peso sumergido) es significativa $(p<0,01)$ y definida por la ecuación:

$$
i_{b}=0,0008 Q^{1.47} \quad\left(r^{2}=0,47\right)
$$

La desviación estadistica de la relación a causa de la dispersión de puntos sobre la recta ha sido calculada en un 65\%. Como en el caso del sedimento en suspensión, esta dispersión es debida a causas de variabilidad estacional asl como a fenómenos de histéresis durante las crecidas.

La producción media anual de sedimento como carga de fondo es de $6643 \mathrm{Tm}$., lo que significa una contribución de $626 \mathrm{~kg} \mathrm{ha}^{-1} \mathrm{an}^{-1}$. La carga de fondo es, por tanto, más de $65 \%$ de la producción anual de material sólido y el $46 \%$ de la producción global de sedimento de la cuenca de Arbúcies. La importancia de la contribución de la carga de fondo a la producción anual de la cuenca es más alta que la publicada en otros estudios en rios de arenas (Lane \& Borland 1951, Simons \& Senturk 1977), asi como para rios de gravas 
MAGNITUD Y FRECUENCIA DEL TRANSPORTE FLUVIAL DE SEDIMENTO

(McPherson 1971, Dietrich \& Dunne, 1978). La elevada producción de sedimento como carga de fondo en la riera de Arbúcies es debida, principalmente, a la alta frecuencia de caudales capaces de transportar grandes cantidades de arena y gravas finas.

\section{Discusión y conclusiones}

A modo de resumen de los resultados de transporte de sedimento en esta cuenca la figura 3 muestra las curvas de frecuencias acumuladas de la carga de material en disolución, en suspensión y de fondo. La carga en disolución es claramente más uniforme y constante en el tiempo que el material sólido transportado en suspensión y como carga de fondo, el cual depende mucho más de las crecidas, especialmente de aquellas asociadas a niveles de cauce lleno o bankfull. Tanto es asi que en el $2,2 \%$ del tiempo se transporta el $56 \%$ de la producción de sedimento en suspensión y el $31 \%$ de la carga de fondo, y en el $20 \%$ del tiempo se transporta el $95 \%$ del material en suspensión y el $78 \%$ de la de fondo. En concreto, la carga de sedimento en suspensión se ve más afectada por los parámetros hidráulicos actuantes en el canal que la carga arrastrada por el fondo, y es por tanto más dependiente de las condiciones del flujo. El transporte de carga de fondo es ligeramente más uniforme y constante a lo largo del tiempo que el sedimento transportado en suspensión.

La relación entre caudal efectivo para el transporte de material sólido en esta cuenca y el caudal geomorfológicamente dominante (bankfull) parece clara en la cuenca de la riera de Arbúcies. Los resultados muestran que el caudal más efectivo para el transporte de sedimento, tanto en suspensión como carga de fondo, es un suceso relativamente frecuente y de una magnitud moderada (siete dias por año), similar al sugerido por Wolman \& Miller (1960) y confirmado por Andrews (1980). De acuerdo con Andrews (1980) el caudal efectivo para el transporte de sedimento tiene una magnitud idéntica con el caudal bankfull. No obstante Pickup \& Warner (1976), Benson \& Thomas (1966) y Nolan et al. (1987) mostraron que el caudal efectivo está claramente situado por debajo del nivel bankfull. Por su parte, Ashmore \& Day (1988) indicaron que el caudal efectivo depende de la superficie de la cuenca y, por tanto, cualquier generalización sobre el tema es complicada. Los resultados obtenidos en la cuenca de Arbúcies no resuelven las aparentes discrepancias en términos de área drenada que se derivan del trabajo de Ashmore \& Day (1988). Los datos de Arbúcies añaden validez al concepto de caudal efectivo para transporte fluvial de sedimento, tal y como fue desarrollado por Wolman \& Miller (1960). Además, estos resultados especifican la magnitud y la frecuencia del caudal efectivo en ambientes Mediterráneos semihúmedos, caracterizados por una escorrentía subsuperficial y por flujos de base constantes, con una variabilidad moderada en el régimen hidrológico, y donde la red de drenaje puede no estar necesariamente ajustada solamente a los sucesos hidrológicos extremos. 
Agradecimientos. Este trabajo ha sido financiado con una Beca de Formación de Personal Investigador (F.P.I.) del Ministerio de Educación y Ciencia, y con ayudas a la investigación de la CIRIT, Generalitat de Catalunya. Agradecemos, asimismo, la colaboración de la Junta d'Aigües, Generalitat de Catalunya, en la instrumentación de la estación de aforos y en las facilidades de acceso a los datos hidrológicos de la cuenca. Parte de este artículo ha sido discutido con el Dr. Marwan Hassan, University of British Columbia. Agradecemos la colaboración técnica del Dr. Peter Ergenzinger, Freie Universität Berlin.

\section{Referencias}

Andrews, E.D. (1980): Effective and bankfull discharges of streams in the Yampa river basin, Colorado and Wyoming. Journal of Hydrology, 46: 311-330.

Ashmore, P.E. \& Day, T.J. (1988): Effective discharge for suspended sediment transport in streams of the Saskatchewan river basin. Water Resources Research, 24 (6): 864-870.

Baker, V.R. (1977): Stream-channel response to floods, with examples from Central Texas. Geological Society of America Bulletin, 88: 1057-1071

Batalla, R. (1993): Contribució del transport de sorres en el balanç de sediment d'una conca granítica Mediterrània. Tesis doctoral. Universitat de Barcelona, $199 \mathrm{p}$.

Batalla, R. \& Sala, M. (1992): Balanç hidroquímic d'una conca mediterrània en estat seminatural: el cas de la riera d'Arbúcies. Acta Geológica Hispánica, 27, n³

Batalla, R. \& Sala, M. (1993): Effective discharge for bedload transport in a subhumid Mediterranean sand-bed river (Arbúcies, NE Spain). En: Hickin,T.J. (ed.) Rivers. John Wiley \& Sons, (en prensa)

Batalla, R. \& Sala, M. (1994): Temporal variability of the suspended sediment transport in a Mediterranean sandy gravel-bed river. En: Olive, L.J. (ed.): Variability in stream erosion and sediment transport. IAHS Publ. Canberra (en prensa)

Beard, L.R. (1975): Generalized evaluation of flash-flood potential: Texas Univ. Center Research Water Resources Tech. Rept. CRWR-124, $27 \mathrm{p}$.

Benson, M.A. \& Thomas, D.M. (1966): A definition of dominant discharge, Bull. Int. Assoc. Sci. Hydrol., 11: 76-80.

Carson, M.A., Taylor, C.H. \& Grey, B.J. (1973): Sediment production in a small Appalachian watershed during spring runoff: the Eaton basin, 1970-1972. Canadian Journal of Earth Sciences, 10: 1707-1734

Dietrich, W. \& Dunne,T.(1978): Sediment budgetfor a small catchment in mountanious terrain. Zeitschrift für Geomorphologie, N.F. Suppl. Bd. 29: 191-206

Emmet, W.W. (1970): The hydraulics of overland flow. U.S. Geol. Surv. Prof. Paper, 662A

Emmet, W.W. (1979): A field calibration of the sediment trapping characteristics of the Helley-Smith bedload sampler. US Geological Survey Professional Paper, 1139.

Ferguson, R.I. (1986): River loads underestimated by rating curves. Water Resources Research, 22 (1): 74-76

Lane, E.W. \& Lei, K. (1950): Streamflow variability. Transactions of the American Society of Civil Engineers, 115: 1084-1134. 
MAGNITUD Y FRECUENCIA DEL TRANSPORTE FLUVIAL DE SEDIMENTO

Lane, E.W. \& Borland, W.M. (1951): Estimating bedload. Transactions of American Geophysical Union, 32(1): 121-123

Leopold, L.B. \& Maddock, T.(1953): The hydraulic geometry of stream channels and some physiographic implications. U.S. Geol. Surv Prof. Paper, 352G

Martin Vide, J. (1985): Estacionalidad de la precipitación y mediterraneidad en el Pirineo Catalán. Notes de Geografia Fisica, 13-14: 57-66

Nolan, K.M., Lisle, T.E. \&.Kelsey, H.M. (1987): Bankfull discharge and sediment transport in northwestern California, Erosion and sediment in the Pacific Rim, IAHS Publ., 165: 439-449.

Pickup, G. \& Warner, R.F. (1976): Effects of hydrologic regime on magnitude and frequency of dominant discharge, Journal of Hydrology, 29: 51-75.

Piest, R.F. (1964): Long-term sediment yields from small watershed. En: Land Erosion, Precipitation, Hydrometry, Soil Moisture. Proc. Berkeley General Assembly of IUGG, IAHS Publ. 65: 121-140

McPherson, H.J. (1971): Dissolved, suspended and bedload movement patterns in Two O'clock Creek, Rocky Mountains, Canada, Summer, 1969. Journal of Hydrology. 12: $221-233$

Sala, M. (1983): Fluvial and slope processes in the Fuirosos basin, Catalan Ranges, north east lberian coast. Zeitschrift für Geomorphologie, N.F. 27: 393-41.1

Sala, M. \& Wheeler, D. (1988): Variación espacial de la carga de sedimentos en la cuenca del rio Tordera en condiciones de estiaje. Acta Geológica Hispánica, 23 (3): 209-216

Schumm, S.A. (1960): The shape of the alluvial channels in relation to sediment type, U.S. Geological Survey Professional Paper, 352-B.

Simons, D.B. \& Senturk, F. (1977): Sediment transport technology. Water Resources Publications, Fort Collins, Colorado, $807 \mathrm{pp}$.

Walling, D.E. (1974): Suspended sediment and solute yields from a small catchment prior to urbanization. En: Gregory, K.J. \& Walling, D.E. (eds.): Fluvial processes in instrumented watersheds. Institute of British Geographers Special Publication, 6 : 169-192

Walling, D.E. (1977): Limitations of the rating curve technique for estimating suspended sediment loads, with particular reference to British rivers. IAHS PubI. 22: 34-48

Walling, D.E. (1984): Dissolved loads and their measurements. En: Hadley, R.F. \& Walling, D.E.: Erosion and sediment yield: Some methods of measurement and modelling. London, Geo Books.

Walling, D.E. \& Webb, B.W. (1981): Water quality. En Lewin, J. (ed.) British rivers. George Allen, pp. 126-169, Boston

Wolman, M.G. (1955): The natural channel of Brandywine Creek, Pennsylvania. U.S. Geological Survey Professional Paper, 271.

Wolman, M.G. \& Miller, J.P. (1960): Magnitude and frequency of forces in geomorphic processes. Journal of Geology, 68: 54-74. 